\title{
Co-designed paleo experiments on land-cover and land-use change impacts
}

\author{
Sandy P. Harrison', M.-J. Gaillard ${ }^{2}$ and B.D. Stocker ${ }^{3}$
}

Sitges, Spain, 24-28 September 2018

\begin{abstract}
Members of the PAGES LandCover6k working group (LandCover6k), the Paleoclimate Modeling Intercomparison Project (PMIP), and representatives of the carbon-cycle modeling community and PAGES' PEOPLE 3000 working group met in Sitges, Spain, to co-design paleo simulations to evaluate the impact of land-use and anthropogenic landcover change on climate and the carbon cycle over the Holocene.
\end{abstract}

The impact of anthropogenic land-cover change due to land use (LULC) on biogeochemical cycles and climate is still uncertain. Climate-model simulations indicate that LULC impacts on temperature and precipitation are large, both in and beyond the regions where these changes occur (Smith et al. 2016). However, the LULC changes used to drive these simulations are unrealistic (Gaillard et al. 2010). Furthermore, the currently available LULC scenarios (HYDE: Klein Goldewijk et al. 2017; KK10: Kaplan et al. 2011) are inconsistent with the constraints imposed by carbon budgeting (Stocker et al. 2017). Creating more realistic LULC scenarios, using paleovegetation reconstructions and archaeological data, is the central goal of LandCover6k.

The group has made considerable progress towards LULC reconstructions for key regions and times (Morrison et al. 2018). It is

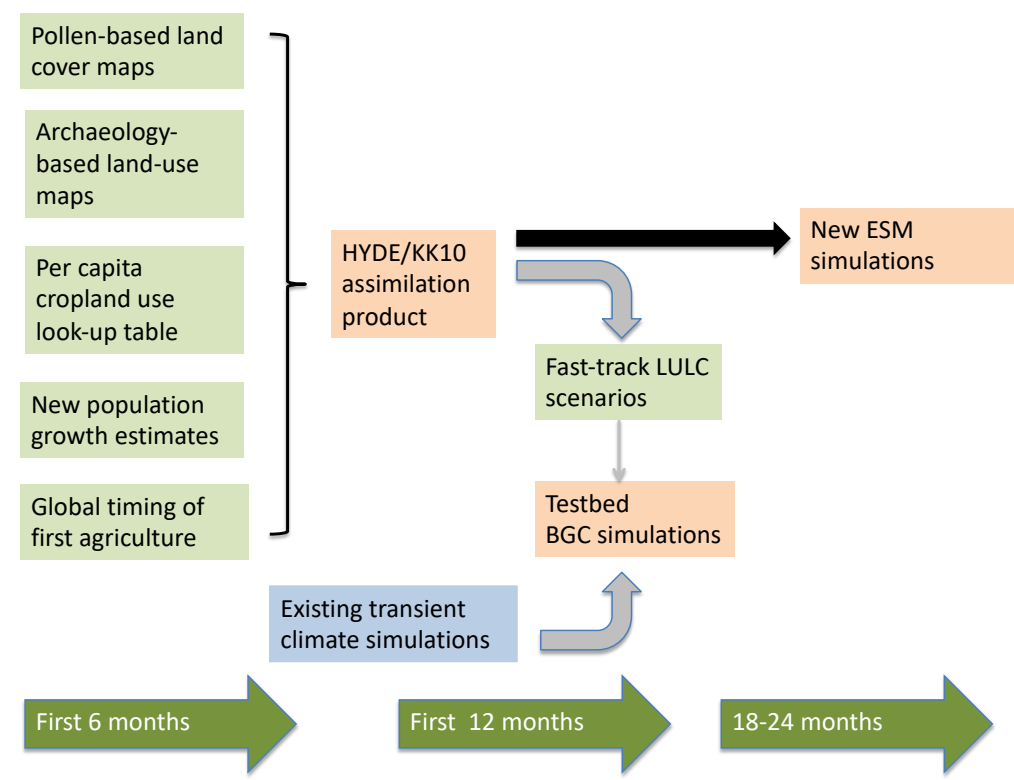

Figure 1: Schematic showing how LandCover6k fast-track products will feed into the production of new land use-land cover (LULC) scenarios. Offline simulations with biogeochemical models will provide a validation of the realism and impact of the LandCover6k products. The ultimate goal is to produce new LULC scenarios as forcing for Earth system model simulations. now time to test and use these reconstructions. Discussions at the Sitges workshop focused on how to incorporate LandCover6k information into LULC scenarios, to design biogeochemical model simulations to test the reliability of these reconstructions, and to design Earth system model simulations to provide a realistic assessment of the impact of LULC changes on climate and the carbon cycle over the Holocene.

The first day of the workshop focused on overview presentations of the various initiatives relevant to this goal and the research interests of different communities (for presentations see the LandCover6k homepage: pastglobalchanges.org/ini/wg/landcover6k/ intro). Discussions around the presentations ensured there was common understanding of the terminology and clarified the data needs of each community. Subsequent breakout group discussions addressed co-operative activities from several different perspectives. Key issues were (a) how information from the LandCover6k project could be used to improve the HYDE and KK10 scenarios or (b) as input to and/or validation of climate model simulations, (c) how HYDE and KK10 scenarios could be used as input for climate model and/or carbon-cycle simulations, and (d) whether existing products fulfilled the minimum and/or desirable , while the longer-term goals include LandCover6k products that will be ready by the end of the second phase of LandCover6k. A more complete description of the experimental protocols will be published as a joint-authored paper, to help enable modeling groups to run the LandCover6k-PMIP co-designed simulations.

\section{AFFILIATIONS \\ 'School of Archaeology, Geography and Environmental Science, University of Reading, UK 2Department of Biology and Environmental Science, Linnaeus University, Kalmar, Sweden ${ }^{3}$ Centre for Ecological Research and Forestry Applications, Bellaterra, Spain \\ CONTACT \\ Sandy P. Harrison: s.p.harrison@reading.ac.uk}

REFERENCES

Crombé P, Robinson E (2014) J Arch Sci 52: 558-566

Gaillard MJ et al. (2010) Clim Past 6: 483-499

Kaplan JO et al. (2011) Holocene 21: 775-791

Klein Goldewijk K et al. (2017) Earth Syst Sci Data 9: 927-953

Morrison KD et al. (2018) Past Global Changes 26: 8-9

Smith MC et al. (2016) Clim Past 12: 923-941

Stocker BD et al. (2017) Proc Natl Acad Sci 114: 1492-1497 